\title{
Interpopulation hybridization results in widespread viability selection across the genome in Tigriopus californicus
}

Victoria L Pritchard ${ }^{1,3^{*}}$, Leilani Dimond ${ }^{1}$, J Scott Harrison ${ }^{2,4}$, Claudia Cristina S Velázquez ${ }^{1}$, Jennifer T Zieba ${ }^{1}$, Ronald S Burton ${ }^{2}$ and Suzanne Edmands ${ }^{1}$

\begin{abstract}
Background: Genetic interactions within hybrids influence their overall fitness. Understanding the details of these interactions can improve our understanding of speciation. One experimental approach is to investigate deviations from Mendelian expectations (segregation distortion) in the inheritance of mapped genetic markers. In this study, we used the copepod Tigriopus californicus, a species which exhibits high genetic divergence between populations and a general pattern of reduced fitness in F2 interpopulation hybrids. Previous studies have implicated both nuclear-cytoplasmic and nuclear-nuclear interactions in causing this fitness reduction. We identified and mapped population-diagnostic single nucleotide polymorphisms (SNPs) and used these to examine segregation distortion across the genome within F2 hybrids.
\end{abstract}

Results: We generated a linkage map which included 45 newly elucidated SNPs and 8 population-diagnostic microsatellites used in previous studies. The map, the first available for the Copepoda, was estimated to cover $75 \%$ of the genome and included markers on all $12 \mathrm{~T}$. californicus chromosomes. We observed little segregation distortion in newly hatched F2 hybrid larvae (fewer than $10 \%$ of markers at $p<0.05$ ), but strikingly higher distortion in F2 hybrid adult males (45\% of markers at $\mathrm{p}<0.05$ ). Hence, segregation distortion was primarily caused by selection against particular genetic combinations which acted between hatching and maturity. Distorted markers were not distributed randomly across the genome but clustered on particular chromosomes. In contrast to other studies in this species we found little evidence for cytonuclear coadaptation. Instead, different linkage groups exhibited markedly different patterns of distortion, which appear to have been influenced by nuclear-nuclear epistatic interactions and may also reflect genetic load carried within the parental lines.

Conclusion: Adult male F2 hybrids between two populations of T. californius exhibit dramatic segregation distortion across the genome. Distorted loci are clustered within specific linkage groups, and the direction of distortion differs between chromosomes. This segregation distortion is due to selection acting between hatching and adulthood.

\section{Background}

One way in which the integrity of species can be maintained is by intrinsic postzygotic barriers which reduce the fitness of hybrid offspring. Understanding how genetic interactions within interpopulation hybrids influence fitness is hence an important step towards understanding how new species arise. When gene flow

\footnotetext{
* Correspondence: victoria.pritchard@noaa.gov

'Department of Biological Sciences, University of Southern California, Los Angeles, California 90089-0371, USA

Full list of author information is available at the end of the article
}

between populations is restricted, they can diverge as a result of selection and drift. One outcome of this divergence may be the accumulation of 'Dobzhansky-Muller incompatibilities', mutations which only become deleterious when placed on a novel genetic background in hybrids between the populations [1,2]. As first generation (F1) hybrids contain a full haploid nuclear genome from each of the parental populations, the negative fitness consequences of Dobzhansky-Muller incompatibilities may not be expressed until the second hybrid (F2 and backcross) generations, when co-adapted blocks of

\section{Ciomed Central}


the genome have been broken up by recombination. Studies have now identified regions of the genome, and in some cases specific genes, involved in such deleterious Dobzhansky-Muller interactions in hybrids [3-6]. In contrast, some authors have observed evidence for favorable interactions between divergent parental genomes in interpopulation and inter-species hybrids [7-10], suggesting that recombination of parental genomes also has the potential to increase fitness. Examination of the patterns of segregation distortion at genetic markers distributed across the genome within hybrids can both help identify candidate regions for Dobzhansky-Muller incompatibilities [11], and provide insights into other types of genetic influence on hybrid fitness.

The harpacticoid copepod Tigriopus californicus, an easily cultivated species that is common in supra-littoral splash pools on rocky outcrops along the west coast of North America, has become an established model for investigating the genetic basis of hybrid breakdown. There is little gene flow between populations, even those separated by relatively short distances [12,13]. Divergence in mtDNA nucleotide sequence between populations separated by less than $60 \mathrm{~km}$ can exceed $20 \%$, which encompasses both synonymous and nonsynonymous sites and reflects both this low gene flow and an unusually high mtDNA substitution rate compared to nuclear genes $[14,15]$. The level of divergence at nuclear loci is still substantial, but is between 5.8 and 38.2-fold lower than mitochondrial divergence between the same geographic locations [14]. Despite such extreme genetic differentiation, almost all populations can easily be crossed to produce viable hybrids $[16,17]$. Interpopulation crosses typically exhibit a pattern of slightly increased fitness in the F1 generation, followed by lowered fitness in the F2 and later hybrid generations, as demonstrated by a variety of measurements including fecundity, development time and survivorship [18-20], response to osmotic stress [21,22], cytochrome oxidase activity [23] and mitochondrial ATP production [22]. This pattern suggests a role for Dobzhansky-Muller incompatibilities in causing the hybrid breakdown. The degree of fitness reduction in F2 hybrids shows a significant association with genetic and geographic distance between populations [16].

Much work has focused on the role of nuclear-mitochondrial incompatibilities in this hybrid breakdown. Ellison and Burton [20] found clear evidence of this intergenomic interaction. They backcrossed F3 hybrid females, which exhibited lowered fitness, to pure males from each parental population: they found that restoring a full nuclear haplotype in this way restored fitness only if the mtDNA from the matching population was also present. At least two sets of candidate loci for nuclear- mitochondrial interactions have attracted investigation. First, there has been much interest in the interaction between nuclear and mitochondrial encoded proteins involved in the oxidative phosphorylation (OXPHOS) pathway. In [24], the authors observed a general trend towards loss of fitness, associated with reduced ATP production, in hybrid lines compared to parental controls. They found that those OXPHOS enzyme complexes that contained both mitochondrial and nuclear components exhibited reduced activity in hybrid lines, whilst those containing only nuclear components did not, implicating nuclear-mitochondrial interactions in generating the observed results. Several studies have found strong evidence for nuclear-mitochondrial coadaptation affecting the activity of cytochrome c oxidase (COX), an enzyme with both nuclear and mitochondrial encoded units, and its interaction with the nuclear encoded cytochrome c $[23,25,26]$. Population specific interactions between COX and cytochrome c have been traced to the level of individual amino acid substitutions [27]. Second, Ellison and Burton [20] have suggested that there is also coadaptation in the mitochondrial transcription apparatus. Mitochondrial RNA polymerase (mtRPOL, nuclear-encoded) initiates mtDNA transcription by binding specific mtDNA sequence in the noncoding "control region." Using inbred hybrid lines, the authors [22] found that lines with mtRPOL and mtDNA from the same population exhibited parental OXPHOS expression patterns, whilst those with mismatching mtRPOL and mtDNA did not.

However, cytonuclear coadaptation is clearly not complete within all $T$. californicus populations [23,28] and evidence suggests that interactions within the nuclear genome also play a role in hybrid breakdown. For example, Ellison and Burton [22] found that, for hybrid lines containing mtDNA from a San Diego population (SD), parental expression was not restored with the matching mtRPOL, suggesting that at least one additional, epistatically interacting nuclear locus is present in this population but not in the experimental lines. Willett and Berkowitz [29] found dramatic segregation distortion for two nuclear encoded homologues of malic enzyme in F2 hybrids, which could be attributed to selection against particular genotypes acting between hatching and sexual maturity. Willett [30] examining segregation patterns of three nuclear-encoded OXPHOS genes, found evidence for complex nuclear epistatic interactions, but not nuclear-mitochondrial interactions, affecting survival to adulthood of F2 hybrids. Edmands and colleagues $[28,31]$ similarly implicated epistatic interactions between different chromosomes in influencing segregation distortion in backcross and F2 hybrids.

In this study, we investigated the genetic composition of $T$. californicus interpopulation hybrids in more detail. 
We made crosses between two populations, SD and SC, that are $640 \mathrm{~km}$ apart and more than $20 \%$ divergent in mitochondrial COI and CYTB sequence $[15,16]$. These populations display the classic pattern of decreased survivorship of F2 interpopulation hybrids compared to parentals (Edmands and coworkers, unpublished data), and have been used in previous studies. We examined segregation pattern of eight microsatellite markers and 45 newly elucidated single nucleotide polymorphism (SNP) markers, distributed over the genome, in the F2 individuals. We compared patterns of segregation distortion between newly hatched nauplii (larvae) and adults in order to distinguish distortion caused by differential post-hatching viability from distortion caused by either meiotic drive, differential gametic fertilization success or pre-hatching mortality.

\section{Methods}

\section{SNP discovery and genotyping}

A cDNA library was constructed from RNA extracted from a mass copepod sample collected from the San Diego population (SD, $\left.32^{\circ} 45^{\prime} \mathrm{N}, 117^{\circ} 15^{\prime} \mathrm{W}\right)$ of $T$. californicus. This sample (approximately $1 \mathrm{~g}$ wet weight) included adults of both sexes as well as eggs, nauplii and copepodid developmental stages. Total RNA was extracted using Tri Reagent (Sigma Chemical) using the manufacturer's protocol. Purification of mRNA from total RNA was achieved using a Qiagen mRNA isolation kit. Approximately $6 \mu \mathrm{g}$ of mRNA was then used to create the cDNA library using the Zap Express cDNA Synthesis Kit (Stratagene), following manufacturer's protocols. Cloned cDNAs were recovered by in vivo excision of the pBluescript ${ }^{\circledR}$ phagemid in E. coli, and 1100 positive clones (based on blue/white screening) were randomly selected for sequencing. Inserts from the positive clones were PCR amplified using M13 primers and then sequenced using T3 and T7 primers. Sequencing was carried out on an Amersham MegaBACE 500 sequencer with Amersham's ET Dye-Terminator chemistry.

From the cDNA sequences, we selected non-mitochondrial sequences over $700 \mathrm{bp}$ in length and designed primers to amplify 500-700 bp portions of these sequences using Primer 3 (http://primer3.sourceforge. net/). For all primer design, we specified an optimal primer length of $20 \mathrm{bp}$ and optimal melting temperature of $60^{\circ} \mathrm{C}$. We also designed primers to amplify three portions of the mitochondrial genome, using mitochondrial sequences for T. californicus deposited in GenBank. Primers were manufactured by Operon Biotechnologies (http://www.operon.com) and IDTDNA (http://www. idtdna.com). We tested each primer pair on two individuals from each of three populations: SD, SC (Santa Cruz, California, 36 $57^{\prime} \mathrm{N}, 1^{\circ} 2^{\circ} 03^{\prime} \mathrm{W}$ ), and PBJ (Punta
Baja, Baja California, $36^{\circ} 57^{\prime} \mathrm{N}, 122^{\circ} 03^{\prime} \mathrm{W}$, used in a separate study). DNA was extracted by placing copepods in $50 \mu \mathrm{l}$ lysis buffer $(10 \mathrm{mM}$ Tris $\mathrm{pH} 8.3,50 \mathrm{mM} \mathrm{KCl}$, $0.5 \%$ Tween 20) with $200 \mu \mathrm{g} / \mathrm{ml}$ Proteinase $\mathrm{K}$ and incubating at $65^{\circ} \mathrm{C}$ for $2 \mathrm{hr}$ followed by $100^{\circ} \mathrm{C}$ for $15 \mathrm{~min}$. We amplified products in $25 \mu \mathrm{l}$ reactions using the following recipe: $2.5 \mu \mathrm{l}$ template DNA; $2.5 \mu \mathrm{l}$ each forward and reverse primers (10 $\mathrm{mM}$ each); $2.5 \mu \mathrm{l}$ premixed dNTPs (2.0 mM each); $2.0 \mu \mathrm{lgCl}_{2}$ (25 mM); $2.5 \mu \mathrm{l} 10$ $\mathrm{X}$ buffer, 10.4 $\mu$ l DNA-free water, $0.1 \mu \mathrm{l}$ Taq polymerase, and the following reaction conditions: a denaturation step of $94^{\circ} \mathrm{C}$ for 5 minutes; 35 cycles of $94^{\circ} \mathrm{C}$ for 30 sec, $55^{\circ} \mathrm{C}$ for $30 \mathrm{sec}, 72^{\circ} \mathrm{C}$ for $30 \mathrm{sec}(45 \mathrm{sec}$ for mtDNA primers), followed by a final extension step of $72^{\circ} \mathrm{C}$ for 5 minutes. PCR products were visualized on $1.8 \%$ agarose gels with ethidium bromide staining. Where a primer pair successfully produced a single amplification band in SD and at least one of the other test populations, the product was sequenced. Products were sent to the High-Throughput Genomics Unit at the University of Washington (http://www.htseq.org) for exo/sap cleanup followed by unidirectional sequencing. Two individuals per population were considered sufficient for preliminary identification of population-specific SNPs as geographically isolated $T$. californicus populations tend to be genetically homogeneous [32]. Levels of polymorphism were subsequently determined by genotyping at least 28 individuals from each of the three populations.

We used Sequencher v. 4.6 (http://www.genecodes. com) to align sequences and searched for SNPs diagnostic between both SD and SC, and SD and PBJ, with $>25$ bp of invariant sequence either upstream or downstream to enable the design of single base extension primers. In addition, we screened $T$. californicus mtRPOL sequence, obtained from GenBank, to identify suitable SNPs between SD and SC and SD and PBJ. We selected one suitable SNP site for each separate nuclear sequence and three from the mitochondrial sequences. SNP and flanking sequence information were provided to Jeffrey Conroy, Genomic Shared Resources, Roswell Park Cancer Institute, Buffalo, NY, who performed primer design using iPLEX Gold software (Sequenom, San Diego, CA).

All SNP genotyping was performed at Roswell Park Cancer Institute, using the iPLEX Gold Assay on a MassARRAY Compact (Sequenom). We provided unpurified, dried-down, lysis extracts $(10 \mu \mathrm{l}$ for adults, $20 \mu \mathrm{l}$ for nauplii). Diagnostic utility of SNPs was confirmed by genotyping a larger sample of copepods from SD, SC and PBJ. Accuracy of SNP calls in heterozygotes was tested by including a blind set of known F1 SD $\times$ SC and $\mathrm{SD} \times \mathrm{PBJ}$ individuals. We also investigated the utility of the SNPs for T. californicus research in general by genotyping them for 13 additional populations, 
ranging from Washington to Baja California (Additional Files), the majority of which have been used in previous studies $[17,32,33]$

\section{Experimental Crosses}

Reproductive biology of T. californicus is well established [33-35]. Males guard immature females by clasping them with their antennae until the female completes her terminal molt; the female is then inseminated and released. Unmated females are therefore easily obtained by separating clasped pairs. Females mate only once and use stored sperm to fertilize multiple clutches of eggs, with each female producing an average of $~ 300$ progeny [35]. Inbred 'isofemale' lines are therefore established by isolating a single fertilized female and allowing offspring, including those from overlapping generations, to mate freely.

Mapping crosses were initiated using isofemale lines from populations SC and SD (one line per population) that had been inbred for 9-10 months (minimum generation time is 23 days at $20^{\circ} \mathrm{C}$ [36]). All crosses were performed in $60 \times 15 \mathrm{~mm}$ Petri dishes containing $10 \mathrm{ml}$ growth medium (1 liter seawater filtered through a 37 $\mu \mathrm{m}$ filter, $0.1 \mathrm{~g}$ ground Tetramin fish food, $0.1 \mathrm{~g}$ powdered Spirulina). One virgin female from the SD line and one adult male from the SC line were placed in each Petri dish. The male was removed when the first eggsac was observed and the female moved to a new Petri dish after each eggsac hatched. The F1 offspring from each clutch were allowed to grow to maturity in the dish. When clasped pairs were observed, they were individually moved to a fresh Petri dish, where they produced the first batch of the F2 generation. Males were removed once an eggsac was observed and females again moved to a new dish after each eggsac hatched. All dishes were housed in a $20^{\circ} \mathrm{C}$ incubator with a $12 \mathrm{~h}$ light: $12 \mathrm{~h}$ dark cycle. Extra Spirulina was added when supplemental food was considered necessary. As crosses were initiated using SD females, all F2 and backcross individuals contained the SD mitochondrial haplotype.

To distinguish between segregation distortion caused by meiotic drive or differential gametic fertilization success, and that caused by post-zygotic selection against particular genetic combinations, we compared results from newly-hatched nauplii to those from adult males. Newly hatched F2 nauplii for genetic analysis were obtained by removing a late-stage (orange-colored) eggsac from a female and allowing nauplii to emerge in a drop of seawater in a Petri dish. Nauplii were then killed by flooding the dish with lysis buffer and individual nauplii were transferred in 2-5 $\mu \mathrm{l}$ of liquid to a $200 \mu \mathrm{l} \mathrm{PCR}$ tube containing $20 \mu \mathrm{l}$ of lysis buffer with $200 \mu \mathrm{g} / \mathrm{ml}$ Proteinase $\mathrm{K}$. Tubes were incubated at $65^{\circ} \mathrm{C}$ for $1 \mathrm{hr}$ followed by $100^{\circ} \mathrm{C}$ for $15 \mathrm{~min}$ and extracts were then stored by freezing at $-70^{\circ} \mathrm{C}$. Adult males were obtained by allowing F2 families to reach maturity in the Petri dish. All males in a dish were rinsed in deionized water and frozen whole in $200 \mu \mathrm{l}$ PCR tubes. DNA was subsequently extracted in $50 \mu \mathrm{l}$ of lysis buffer with $200 \mu \mathrm{g} / \mathrm{ml}$ Proteinase $\mathrm{K}$ using the protocol described above. Genotyped nauplii $(n=190)$ were the offspring of four different F1 pairs; genotyped adult males $(n=205)$ were the offspring of 25 different F1 pairs.

Resources limited genotyping to only one of the two reciprocal crosses and to adults of only one gender (males). Males were chosen to eliminate the possibility of amplifying sperm or zygote alleles in fertilized females. Tigriopus californicus lacks heteromorphic sex chromosomes [37,38] and sex determination, although still poorly understood, may involve both additive genetic and environmental components [39,40]. Previous studies of this species have found differences between the sexes in segregation distortion [25,30,31,41], most commonly finding less distortion in males; however these differences are not systematic, varying with the populations used, direction of cross, locus and replicate. We acknowledge that focusing only on adult males limits the scope of data interpretation; as the first genomewide assessment of larval and adult F2 genotype frequencies in this system, our study nevertheless offers important insight into the mechanism driving segregation distortion.

As female T. californicus do not undergo chromosomal recombination [37], we were also able to produce non-recombinant backcrosses (NR-BC) to confirm that identified linkage groups were on different chromosomes. F1 females were backcrossed to SC males. As before, NR-BC families were allowed to develop to maturity in Petri dishes and adult males $(\mathrm{n}=39$, the offspring of seven different pairs) frozen prior to genetic analysis.

\section{Microsatellite genotyping}

In addition to the SNPs, we genotyped F2 and NR-BC individuals for nine microsatellite loci $(30,197,228,480$, $558,1202,1203,1555,56 \mathrm{~J} 2[42])$ that exhibited fixed size differences between the SD and SC populations in a test sample $(\mathrm{n}=24$ each). These markers enabled SNP linkage groups to be anchored to eight of the $12 \mathrm{~T}$. californicus chromosomes numbered by Harrison and Edmands [31]. Microsatellites were amplified individually in $12 \mu \mathrm{l}$ reactions containing: $0.5 \mu \mathrm{l}$ template DNA; $0.3 \mu \mathrm{l}$ fluorescently labeled forward primer $(10 \mathrm{mM}) ; 1$ $\mu \mathrm{l}$ reverse primer $(10 \mathrm{mM}) ; 1.2 \mu \mathrm{l}$ premixed dNTPs $(2.0$ $\mathrm{mM}$ each); $1.2 \mu \mathrm{l} \mathrm{MgCl}_{2}(25 \mathrm{mM}) ; 1.2 \mu \mathrm{l} 10 \mathrm{X}$ buffer, $6.04 \mu \mathrm{l}$ DNA-free water, $0.06 \mu \mathrm{l} \mathrm{Taq}$ polymerase, with the Thermocycler conditions as described for SNP discovery above (annealing temperature for $480=61^{\circ} \mathrm{C}$, for 
all other microsatellite loci $55^{\circ} \mathrm{C}$ ). PCR products were multiplexed into two pools and run on a CEQ 8000 capillary sequencer (Beckman Coulter, Fullerton, CA, USA). Allele sizes were scored manually.

\section{Statistical Analysis}

Separate linkage maps for adults and nauplii were generated using Map Manager QTX, using the Kosambi map function with linkage criterion set at $\mathrm{p}<0.001$. We estimated corrected map length (L) and coverage (c) using the methods of [43] and [44] as follows. For each linkage group, we added two cM to the total length and then multiplied this by $(m+1) /(m-1)$, where $m$ is the number of markers on each group. We then estimated genomic coverage as $\mathrm{c}=1-\mathrm{e}^{-2 \mathrm{dn} / \mathrm{L}}$ where $\mathrm{d}=$ mean intermarker distance and $\mathrm{n}=$ total number of markers assigned to linkage groups. To examine segregation distortion in nauplii and adults, we compared observed single-locus genotype frequencies to those expected from Hardy-Weinberg equilibrium using the $\chi$-squared goodness-of-fit test. Additionally, we investigated whether pairwise epistatic interactions were contributing to segregation distortion by looking at the co-occurrence of genotypic classes at different SNP loci. For each pair of physically unlinked loci we calculated expected frequencies of each genotypic combination (SDSD/SDSD; SDSD/SDSC; SDSD/SCSC; SDSC/SDSC; SDSC/SCSC; SCSC/SCSC) from the observed frequency of each genotype at each locus. We then compared observed and expected frequencies of genotypic combinations using a $\chi$-squared goodness-of-fit test. We also used $\chi$-squared tests to examine chromosome-level segregation distortion and pairwise linkage disequilibrium between chromosomes in the small non-recombinant backcross sample.

\section{Results}

We generated over 20,000 bp of sequence data for each of the three $T$. californicus populations included in the SNP discovery step. Mean observed nuclear sequence divergence between SD and SC, and between SD and PBJ, was $3.8 \%$, and $3.5 \%$ respectively. Sequence data was submitted to NCBI GenBank (Additional file 1, Table S1). We designed assays for 51 nuclear SNPs and three mitochondrial SNPs (Additional file 1, Table S1), which could be multiplexed into two pools for iPLEX Gold genotyping. Of these, 49 SNPs were identified as diagnostic between SD and SC. On the basis of results from F1 individuals, we rejected a further four SNPs from this study because SD-SC heterozygotes were undercalled, leaving 45 nuclear markers (Additional file 1, Table 1). The majority of SNPs could be scored in all but one of 14 additional T. californicus populations, and generally appeared fixed in each population (Additional file 1, Table S1). However, we note that most of our assays were optimized for just two alternative SNPs, meaning that the presence of additional undetected SNP alleles that did not occur in our three original populations cannot be ruled out: $2 \%$ of the total non-indel polymorphic sites observed in our sequence data exhibited three alleles. Half of all SNPs failed in PA, the only population known to exhibit reproductive isolation from others [17].

One microsatellite (1202) was discovered to be nondiagnostic between SD and SC and was not included in the linkage map. Parents of the non-recombinant backcross individuals carried distinct alleles at this locus and therefore we were still able to use it to anchor one linkage group to a named chromosome.

For both nauplii and adults, nuclear SNPs and microsatellites formed 11 linkage groups and one unlinked locus, corresponding to the 12 chromosomes of T. californicus (Figure 1) [37]. All linkage relationships were strongly supported, with all LOD scores greater than 11 and most much higher (Additional file 2, Table S2). However LOD scores between microsatellites markers and SNP markers were generally lower than between SNP pairs, reflecting the fact that fewer individuals were successfully genotyped for microsatellite loci (mean total $\mathrm{n}$ for SNP loci = 389; for microsatellite loci $=298$ ). Results from non-recombinant backcross individuals confirmed each linkage group to be on a separate chromosome. Inclusion of the microsatellites enabled eight chromosomes named by Harrison and Edmands [31] to be identified (Figure 1). Total length of the nauplii linkage map, calculated by summing all inter-marker distances, was 266.7 cM Kosambi, with a mean betweenmarker distance of $6.5 \mathrm{cM}$ and a maximum distance of $21.2 \mathrm{cM}$. Total adult map distance was very similar at 263.4 cM, however length differences were present between homologous linkage groups in the two maps, reflecting increased segregation distortion in the adults. Most markers were co-linear between the two maps. The greatest variation was caused by the shifting of two microsatellite markers, which may be an artifact of the smaller sample size for this marker type. Maps generated using the SNPs alone (Additional file 2, Table S2) were highly congruent, with the exception that marker TC085 was not assigned to a linkage group in the nauplii. Including the microsatellites, corrected map length for the nauplii was $484.8 \mathrm{cM}$, and estimated genomic coverage for this map was $75.2 \%$. Corrected lengths for other maps are provided in Additional file 2, Table S2.

We observed little segregation distortion in F2 nauplii (Figure 2). Following Bonferroni correction for multiple tests, only one marker (TC078, on Chromosome 8) deviated significantly from Hardy-Weinberg equilibrium, with a deficiency of heterozygotes. We also observed 
Table 1 Name, pre-amplification forward and reverse primers, extension primers and nucleotide calls for the 45 SNP loci used in this study

\begin{tabular}{|c|c|c|c|c|c|}
\hline Name & Forward Primer & Reverse Primer & Extension sequence & SD & SC \\
\hline TC006 & GGGTATAGGGTTTGATCAAC & TTGAGGACCTITTACGCAAC & AAGACCTGTGAAGAA & G & $\mathrm{T}$ \\
\hline TC008 & GGTTGATTGATTITGCCTCC & TGGAGTGCAAAAGTCCATCG & GCCTCCATAGGCTCC & C & $\mathrm{T}$ \\
\hline TC011 & ATGAAAGACTCTTAAGTCG & GATGTCTCACAAATCTCGCC & ACTCTTAAGTCGACAGAC & $\mathrm{T}$ & $A$ \\
\hline TC012 & GCAAACAGGACCGTGTTGAA & ACAAGATGGAACGGGTAGAG & CGTACACCTTGATCTTGCC & C & G \\
\hline TC016 & GTGAGTTTGTCTCAAACGG & ATTGCCAAGCCAAGTAAGCG & TTGTCTCAAACGGTTCACCCA & G & A \\
\hline TC017 & ATGCTTGGCAATTTGGAGTG & TITACCTCCAGGACATTGGC & GCAATTTGGAGTGTTCTGA & C & $\mathrm{T}$ \\
\hline TC033 & TCCCCAAGAGCAATAATCGG & GATCGAAGTITTGGTAGGTC & TGAGAAAATCTCCTACTTACC & C & G \\
\hline $\mathrm{TC} 040$ & GAGGAAGTAGAGCCAATAGC & ACCACGATGGAAGTTGCTTG & CCAATAGCAACCATCA & C & $\mathrm{T}$ \\
\hline TC043 & GCATTGAGGATTGTGGCTTC & TGAGGAAAAATCACGTCCGC & ATTGTGGCTTCACACCTA & C & $\mathrm{T}$ \\
\hline $\mathrm{TC} 045$ & ATTGGGATCCGACCCTGTTG & GGTTGAACACCTGAAATGG & GGGATCTGGTTGTC & $\mathrm{T}$ & C \\
\hline TC046 & GGGAAAACGGGCTCAAGATG & AAAGCTGCCCTTGTAAACGC & ACAAATTGGCTTTGCTTGG & C & $\mathrm{T}$ \\
\hline $\mathrm{TC} 051$ & TCAAGCGAAACACCACCATC & TCGTACACCTGAATGGTGAC & СTTCACCACCTACTC & C & $\mathrm{T}$ \\
\hline TC060 & GAGGACCCATTITTCCACAC & TCTITTACAAGCACTCCTC & GAAGTCATCACACACG & G & A \\
\hline TC073 & ACTTGGATGTGGTGTCCTTG & CCAACACCAATGTCACCATC & GTGGTGTCCTTGGCCTCA & $\mathrm{T}$ & A \\
\hline TC074 & TTGCTCAAGTCAACCATGCC & GCAACCAGTTGATAGCTTCC & TCCTGTATTTTGCCT & C & G \\
\hline TC077 & GTCTCAAACGCGAATGCAAG & TTGCTTGGTTCGAGGACATC & AGAAGCCACGGCCACGTITC & $\mathrm{T}$ & C \\
\hline TC078 & GGGTACTGTGGTGTCGATTC & AAGAGGACAGCAAATGGGAC & TGTCGATTCTCCTCGG & C & G \\
\hline TC084 & GCAAATTCTGGTCCACCATC & ATGCACGTTAATGGCTACCG & TTTATCCGTACTGGTCTC & $\mathrm{T}$ & C \\
\hline TC085 & GAAGCCGACATCAAAATGAC & AAGGACAAATCTCCCATTGC & CATCTGAACCAGAAATCCATC & C & A \\
\hline TC099 & TAGCAATCTTCGGTCACCAC & CAGCCCATTATGATTGCTCG & GGGTTTCCACGGGATA & $\mathrm{T}$ & C \\
\hline TC102 & GCGTCAACGATGTTGAGAAC & TGGTGCCCATACATGAGTTC & GAACTTCATGGCCTTGAAGAT & A & $\mathrm{T}$ \\
\hline TC103 & GAGATCTCAGGAGAGTTGC & AATGAGGAATCTTCTCTGGC & GAGTTGCAACATCTC & $\mathrm{T}$ & C \\
\hline TC104 & GCTTACTTCATTCACGCACC & GAGACTTTGAGCGTCTGAAC & CCATTATTTAGTCTATCCAAGA & A & G \\
\hline TC106 & CATTTCTTCTCCCACACCAG & AATGAGAAGCAATGCTCCTG & ACGGGGATCTTGAC & C & $\mathrm{T}$ \\
\hline TC107 & CCTTCCCATAAAGGTCAATC & TGGAAAGAAACGGGAAGTGC & AAAGGTCAATCAATGACT & C & A \\
\hline TC111 & AAGTGTCTCTGCGAGTGCTG & TTCGAATGATGGGATCCGTG & CAACCTTCGTCGGATGTTCCCGG & C & G \\
\hline TC112 & TGGCTGCCTITGAAGAGAAC & GAAACATCAAAGCAAGCAGG & AGAGAACATCACCTAA & C & $\mathrm{T}$ \\
\hline TC118 & TCAAGGACACCATCAAGAGC & TCCGGACAAGATGTTCTTGC & AGAAGGACGCCTCCTA & $\mathrm{T}$ & C \\
\hline TC124 & ACACCAAACCATTGAGTGGC & ATATGTAGAAGACCGAGAGC & TACCCTGTTGATGCGA & C & $\mathrm{T}$ \\
\hline TC125 & TTGTTGGACTTGATGCCTCG & GCTCGTATTGCTCGATCTTG & ATGTGGACAAATTGAA & G & $A$ \\
\hline TC128 & TGTCGTATCTCGTCCTCTTC & AGAACACGAGAAGAAATTC & CTCGTCCTCTTCTTGGAATC & G & A \\
\hline TC130 & ACGTTCGTITGTTAAGGCGG & GAACATTGCTGACAACGGAG & GTTTGTTAAGGCGGTAACTG & G & A \\
\hline TC152 & CGCCCCAGACGACGACGA & ATGCATTCGCGAAAGGCCTG & СTCCCTCAAAGCCTC & A & G \\
\hline TC155 & TTGGATCAGATCCAGCGGT & GCGAGGATTTGCAAGTACAC & AGATCCAGCGGTGCCTTAAACC & A & C \\
\hline TC156 & AATTTGATCTAGGAGCAACG & GCATTCAGTTCCAGTTTGAG & GGAGCAACGTTGCATTG & C & $\mathrm{T}$ \\
\hline TC157 & CAAGTTCCTGGCCAAGAAAG & TCCATGAACATGTCCATCT & GGGAACGATGATITGGAA & G & C \\
\hline TC162 & GAAGATGCTTGAGTGGATGG & TCGGCGGAGGTGTACACTIT & AAGGCAATCTCCAGGAT & $\mathrm{T}$ & C \\
\hline TC167 & TGGCATCTTGATGTITTCGC & ACTGAGGAAGGATCTTGAGG & AGGTTGGTCATTGT & $\mathrm{T}$ & C \\
\hline TC169 & GTAGCCCCTCAAGGTTATGC & TCACGCTTGGCAACATGGAG & AAGGGCCAATATGTGGC & $\mathrm{T}$ & C \\
\hline TC171 & ATGTCTCAAAACGGCCTTCC & TCGAGGAAGTTTCGTTGCAC & GCCTTCCGGATCAGCGGTA & $\mathrm{T}$ & C \\
\hline TC180 & AATGTCTCGCAAGAGCTGG & TGGTGGTTACCTITCGAAAT & GAGCTGGACCCAGACAC & $\mathrm{T}$ & C \\
\hline TC184 & CTCCCGAATATCATGAAGGC & GGGTCCTCAACGGAAATAAC & AACCCTCAGATTTGGGAGT & G & $\mathrm{C}$ \\
\hline TC188 & GGTCATTCTTCGAGACCTAC & CGATACTIITTGAGAGTAGG & ACTCAACATGTCTGT & G & A \\
\hline TC189 & AGACGTTATGAAGCACGACC & TTGTTATCTTCCAAGGGC & GCACGACCCAATCCGTGTC & $\mathrm{T}$ & A \\
\hline RPOL & CACGTTCATTCAAAGCGGAC & AATCCAAGATTTCAACCTC & GGTTGGTAAGTCGG & G & $\mathrm{T}$ \\
\hline
\end{tabular}

Abbreviations refer to the following T. californicus populations: SD: Point Loma, San Diego, California (32 ${ }^{\circ} 45^{\prime} \mathrm{N}, 117^{\circ} 15^{\prime} \mathrm{W}$; $\mathrm{n}=28$, SC: Santa Cruz, California $\left(36^{\circ} 57^{\prime} \mathrm{N}, 122^{\circ} 03^{\prime} \mathrm{W} ; \mathrm{n}=28\right)$. Additional file 1 , Table S1 provides information on additional SNP loci and SNP calls for additional populations. 


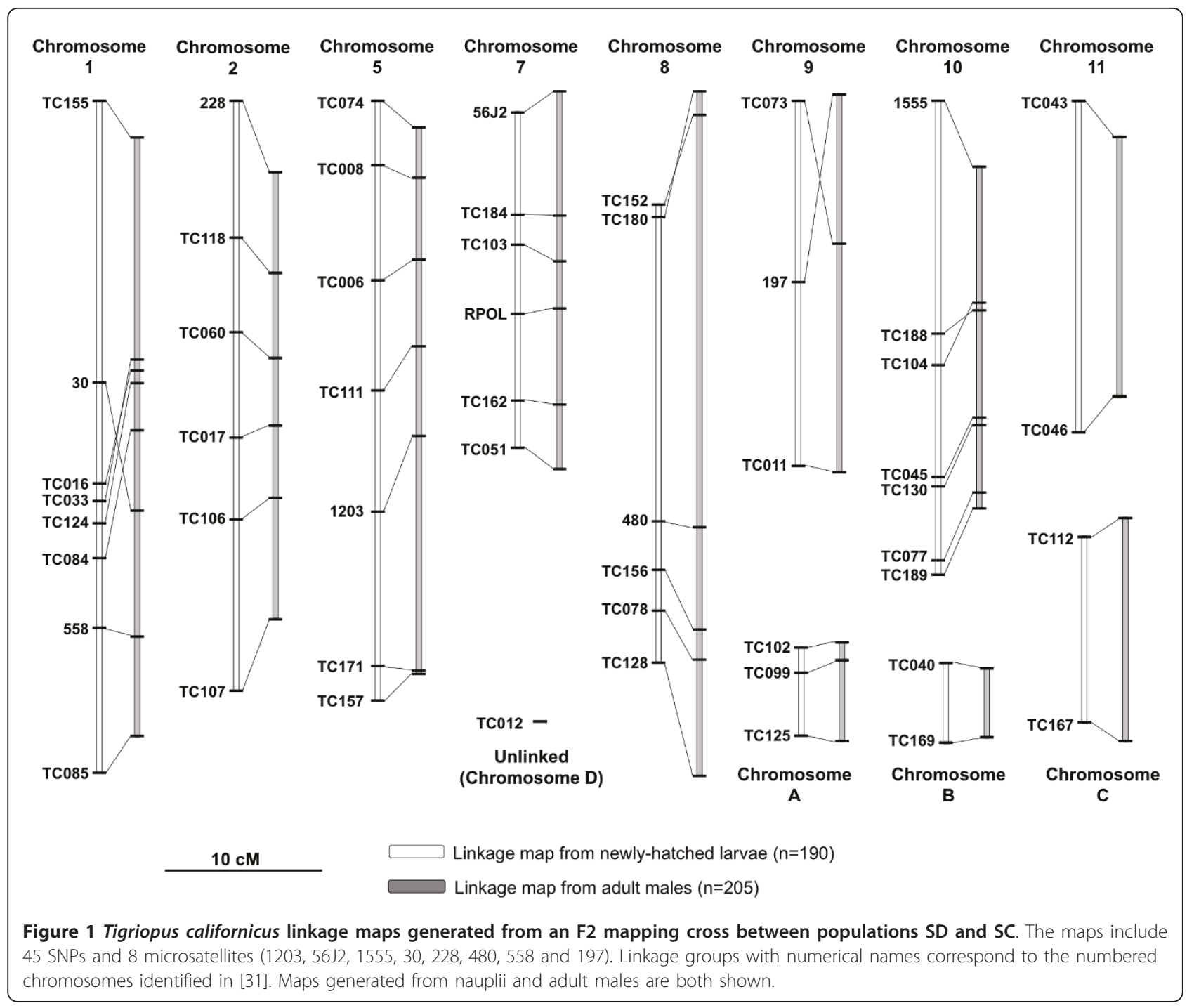

some heterozygote deficiency at the linked marker TC156 and a deficiency of SC homozygotes at two markers on Chromosome 1. The observed deficiency of heterozygotes at microsatellite 1555 may be a technical artifact, due to a large peak size difference between the $\mathrm{SC}$ and SD alleles, in combination with generally lower peaks in nauplii compared to adults, causing heterozygotes to be under-called. Overall in nauplii we observed significantly more SD alleles than expected with a 1:1 ratio assuming equal genetic contributions from both parental lines $\left(\chi^{2}=21.3, \mathrm{p}<0.001\right)$.

In contrast, we observed a high level of significant segregation distortion in adult males, distributed non-randomly across the genome and biased in different directions on different chromosomes (Figure 2). Following Bonferroni correction, eleven markers deviated significantly from Hardy-Weinberg equilibrium. All markers on Chromosome 10 exhibited a very strong deficiency of SD homozygotes. There was also a deficiency of SD homozygotes, associated with an excess of SC homozygotes, at TC011 on Chromosome 9. In contrast, markers on Chromosomes 2 and 7 exhibited an excess of heterozygotes, associated with a deficiency of SC homozygotes. We also observed a deficiency of SC homozygotes and an excess of SD homozygotes at Chromosome B, although this was not significant at the Bonferroni corrected $\mathrm{p}$ value. In adults, we observed overall fewer SD alleles than expected with a 1:1 ratio $\left(\chi^{2}=\right.$ 21.3, $\mathrm{p}<0.001$ ).

We observed four deviations from expected two-locus multiplicative genotypic frequencies between physically unlinked loci at $\mathrm{p}<0.05$. Although it is unclear at what level to correct for multiple testing in this case [45], as comparisons involving different physically linked markers are not independent, we note that none of these deviations were significant after a Bonferroni correction 


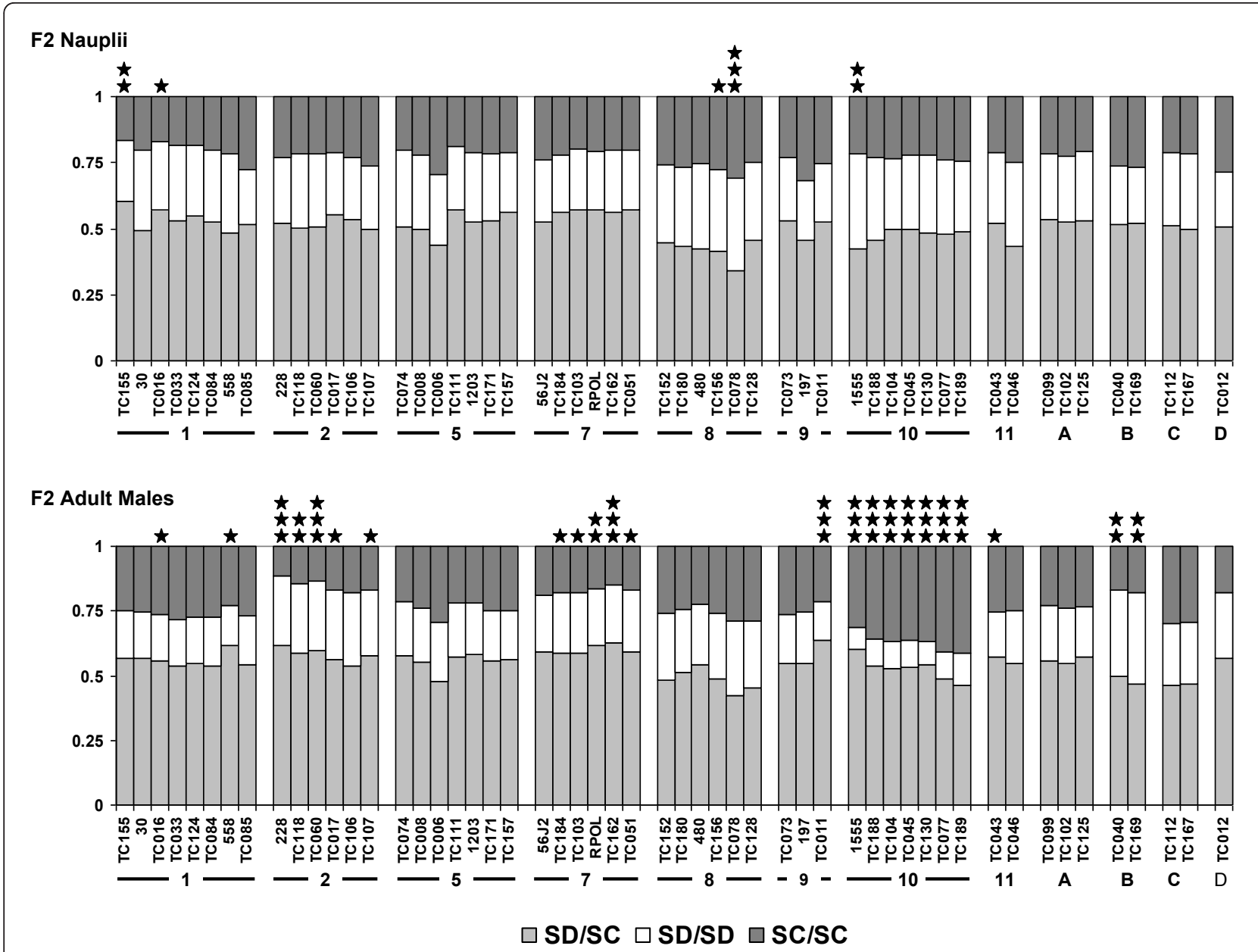

Figure 2 Proportions of heterozygotes, SD homozygotes and SC homozygotes observed at each marker locus for (i) newly hatched nauplii and (ii) adult males. The cytoplasmic background for all individuals was SD. Significance of deviations from expected 2:1:1 Mendelian segregation ratios is indicated by: $\star \star \star p<0.00094$ (Bonferroni corrected $p=0.05$ ); $\star \star p<0.01 ; \star p<0.05$. Markers are arranged by linkage group, as indicated under chart.

based on the number of possible comparisons between linkage groups (132). Three of these deviations involved marker TC167 on Chromosome C and markers on Chromosome 7 (TC 162, TC 184 and RPOL, Figure 3). Both individuals homozygous for SD and those homozygous for SC at Chromosome 7 markers exhibited a strong deficiency of SD homozygotes and a less marked excess of SC homozygotes at marker TC167. Those heterozygous at Chromosome 7 markers showed an excess of SD homozygotes and a deficiency of SC homozygotes at TC167. The additional significant deviation was between marker TC012 on Chromosome D and TC017 on Chromosome 2. Individuals homozygous for $\mathrm{SC}$ at TC012 exhibited a deficiency of SD homozygotes and an excess of heterozygotes at TC017; those homozygous for SD at TC012 showed an excess of SD homozygotes and a deficiency of heterozygotes at TC017 $\left(\chi^{2}=17.7, \mathrm{p}\right.$ $=0.02$ ).
In the non-recombinant backcross, several chromosomes exhibited deviations from the expected 1:1 ratio of homozygotes (SCSC) to heterozygotes (SCSD), however only one (Chromosome 10) was significant following Bonferroni correction, probably reflecting the small sample size (Figure 4). Chromosome 10 exhibited a dramatic deficiency of heterozygotes; in contrast, heterozygote excess was observed at Chromosome 7 and Chromosome B. We found no evidence for epistatic interactions between chromosomes.

\section{Discussion}

In this study, we mapped 45 newly developed SNP markers, and eight pre-existing microsatellite markers onto the genome of $T$. californicus. The map includes markers on all $12 T$. californicus chromosomes and has an estimated genomic coverage of $75 \%$. This is the first linkage map to be generated for the Copepoda, one of 


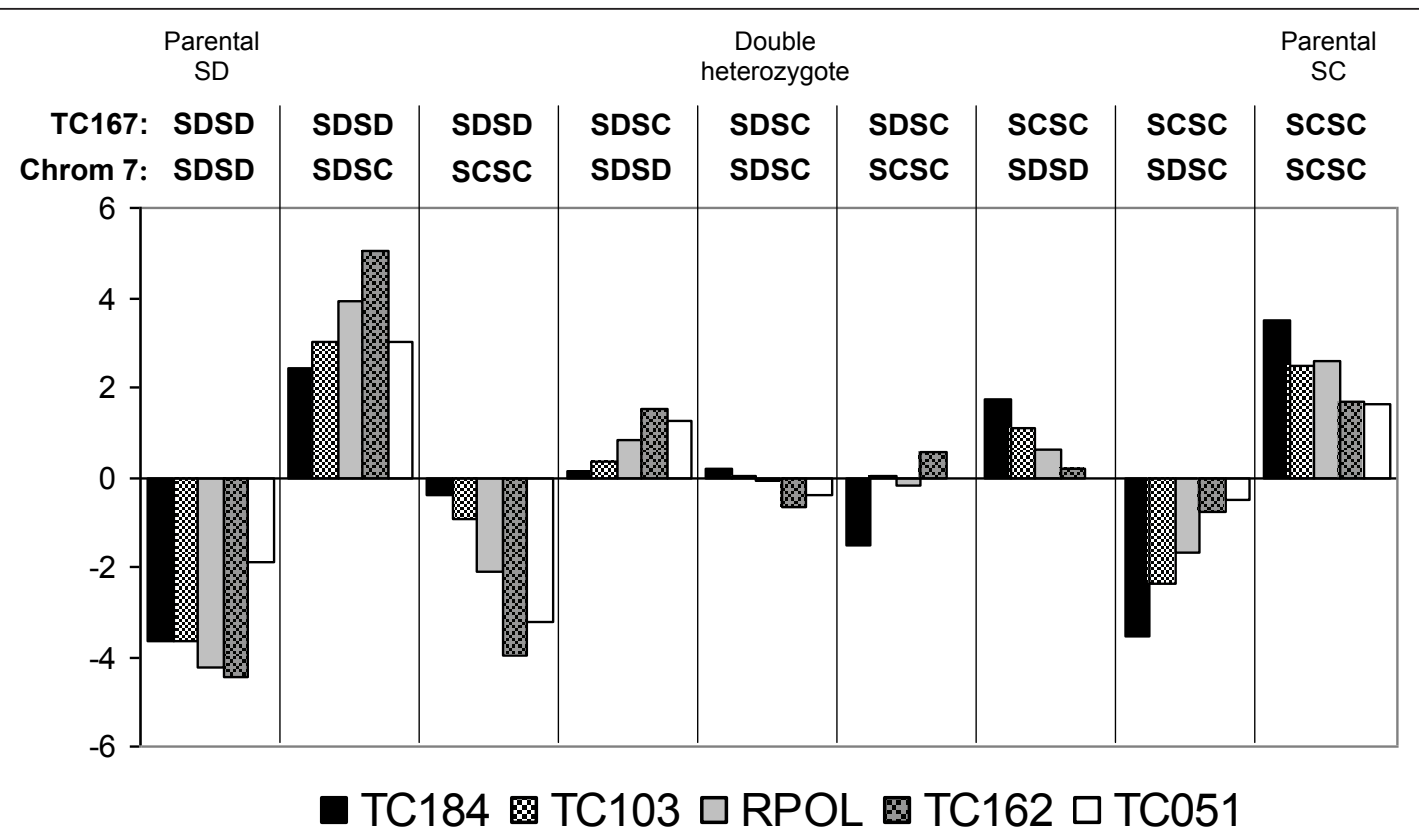

Figure 3 Deviations from expected two-locus genotypic frequencies for marker TC167 and the five markers on Chromosome 7. The y axis indicates the contribution of each possible genotypic combination, as shown above the chart, to the total $\chi^{2}$ value. Negative value indicate that a genotypic combination was observed less frequently than expected by chance, while positive values indicate that a genotypic combination was observed more frequently. Total $\chi^{2}$ values were as follows: TC167 - TC184, $\chi^{2}=17.1, p=0.03 ;$ TC167 - TC103, $\chi^{2}=14.5, p=$ 0.07; TC167 - RPOL, $\chi^{2}=16.2, p=0.04 ;$ TC167 - TC162, $\chi^{2}=18.3, p=0.02 ;$ TC167 - TC051, $\chi^{2}=11.9, p=0.16$.

the most abundant animal taxa on the planet [46], and one of few currently available for crustaceans. All SNP markers are within coding loci, which will facilitate future investigations of chromosome synteny between $T$. californicus and other taxa.

We used this map to investigate patterns of segregation distortion across the genome of F2 hybrids between two T. californicus populations. We observed some

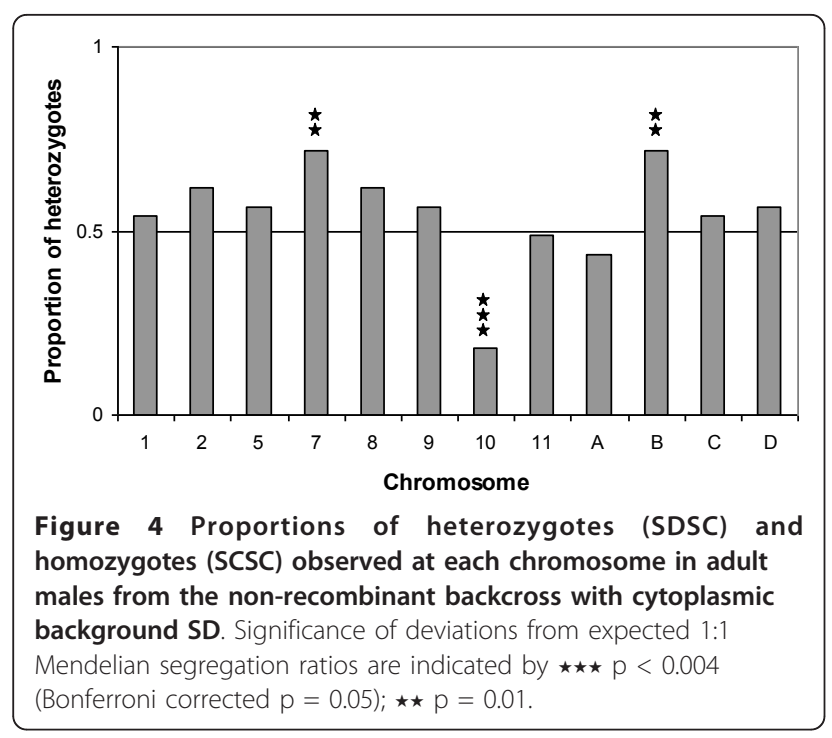

segregation distortion in newly hatched larvae, at fewer than $10 \%$ of markers at $p<0.05$, which may be caused by pre-zygotic effects or pre-hatching mortality, and was not maintained until adulthood. Segregation distortion, however, was strikingly higher in adult males, affecting $45 \%$ of marker loci at $\mathrm{p}<0.05$. This is comparable to the amount of segregation distortion that has been observed within interpopulation or inter-species crosses in other taxa (e.g. Nasonia spp, $29 \%$ of markers in adult males [45]; Mimulus guttatus $48 \%$ of markers in mature plants [47]; Arabidopsis lyrata, 50\% of markers [48]; Daphnia magna, 33\% of markers [44]; Daphnia pulex, $21 \%$ of markers [49]; Lepomis spp. 36.8\% of markers in fry [50]). As seen in other studies, distorted markers were not distributed randomly across the genome but clustered together on particular linkage groups. In $T$. californicus, this clustering may have been exacerbated by the lack of recombination in females, which increases the likelihood that markers physically linked to regions selected against in hybrids will be inherited together.

Our observation of high segregation distortion in F2 hybrid adults but not in F2 nauplii mirrors the pattern seen for several coding loci in different $T$. californicus interpopulation crosses (ME1, ME2 [29]; CYC, RISP, CYC1 $[30,41])$. This suggests that, in general, observed deviations from Hardy-Weinberg equilibrium in interpopulation T. californicus crosses are the result of selection 
against genotypes between hatching and adulthood, rather than being due to meiotic drive or differential gametic fertilization success. Although segregation distortion in inter-species and interpopulation hybrids is a common finding, our study is one of rather few to explicitly demonstrate a role of postzygotic selection in generating this phenomenon. Launey and Hedgecock [51] showed that segregation distortion in the oyster Crassostrea gigas is caused by post-hatching mortality of individuals homozygous for deleterious recessives. Martin and colleagues [52] identified hybrid genetic combinations affecting survivorship in Iris. Rogers and Bernatchez [53] found evidence for selection against particular genotypic combinations acting between fertilization and hatching in backcross hybrids between lake whitefish (Coregonus clupeaformis) ecotypes. Niehuis and colleagues [45] found evidence that cytonuclear co-adaptation caused genotypic-specific mortality between hatching and adulthood in F2 interspecific hybrids in the wasp Nasonia.

While we have data for only one of the two reciprocal crosses, our T. californicus results show very little evidence for cytonuclear coadaptation. All F2 individuals had an SD mitochondrial background: while we did observe an overall excess of SD alleles in nauplii, this had become an overall deficiency of SD alleles in adulthood. In adults we found only two markers (on Chromosome B) where the homozygote matching the SD homozygote was favored, and eight markers where the pattern of segregation distortion was opposite to that which would be expected if the nuclear and mitochondrial genomes within the two populations were coadapted. For example, we observed dramatic segregation distortion in adult F2 males throughout Chromosome 10 , with the direction of segregation distortion indicating strong selection against the nuclear genotype, SDSD, that matched the mitochondrial background. We also observed a large excess of SCSC homozygotes at Chromosome 10 in the non-recombinant backcross, suggesting that this genotype is more fit than the alternative, SDSC. Harrison and Edmands [31] correspondingly observed a deficiency of SDSD Chromosome 10 homozygotes in males (but not in females) in backcrosses between SD and another population, RP (Royal Palms). Taken together, these results suggest that part or all of Chromosome 10 derived from the SD population has a deleterious effect on viability in males of this interpopulation cross, that appears to act in an incompletely dominant manner. There are several reasons why such an apparently deleterious portion of the genome may be maintained in the SD population. First, this deleterious effect may be only expressed in a hybrid nuclear genetic background. Our study did not detect epistatic interactions involving Chromosome 10, although this may be due to limited power. Second, as we only examined males, it is possible that aspects of SD Chromosome 10 may be advantageous in females. Alternatively, genes on SC Chromosome 10 may cause masculinization; we note, however, that while F2 offspring in this study did not deviate from a 1:1 sex ratio, the concurrently generated nonrecombinant backcross was significantly female biased despite all individuals containing at least one copy of the SC chromosome (V.L. Pritchard and coworkers, unpublished data). Third, the deleterious aspect of Chromosome 10 may not be expressed in the natural environment. It may be masked in the wild SD population by the presence of a more dominant allele that was lost both from our isofemale lines and the SD parental line used in [31]. Even if this is not the case, previous studies with $T$. californicus have shown varying experimental conditions to alter the viability of different hybrid genotypes $[26,27,54]$. Additionally, the outcome of replicated experimental hybridizations may vary even under apparently identical conditions [41], suggesting that even apparently minor environmental changes can have a large influence on the fitness of different genotypes. Finally, T. californicus populations in the wild experience repeated population bottlenecks, which are expected to affect the outcome of selection. Hence even if the deleterious aspect of Chromosome 10 is expressed in the natural environment it may persist in the wild SD population due to drift. Indeed, there is evidence that many T. californicus populations carry such a genetic load [28]. For the SD population in particular, previous studies have indicated a selective disadvantage to SD homozygotes for the coding loci ME2 and CYC, even on the SD mitochondrial background [29,41]. In contrast, SD homozygotes were favored on a mismatching mitochondrial background, for the coding loci ME1 and RISP $[29,41]$. We note, however, that these results vary by sex and study, and none of these four coding loci are on linkage groups exhibiting significant segregation distortion in the current cross (ME1, Chromosome C; ME2, Chromosome A; CYC, Chromosome D; RISP, Chromosome 8, Rose and Edmands, unpublished data). Studies of taxa other than T. californicus have also shown that homozygotes mismatching the cytoplasmic background can be favored in hybrids. Fishman and colleagues [55], for example, examining segregation distortion in Mimulus F2 hybrids, found a strong excess of $M$. guttatus homozygotes on a $M$. nasutus cytoplasmic background. Similarly Martin and colleagues [52], in a backcross study using Iris brevicaulis and I. fulva, found that, at three QTLs, presence of I. fulva homozygotes decreased long-term survivorship despite a matching cytoplasmic background.

In contrast to the pattern observed for Chromosome 10, we observe heterozygote excess, with no apparent selection against SDSD homozygotes, throughout most 
of Chromosome 2 and Chromosome 7. We also observe an excess of heterozygotes for Chromosome 7 in the nonrecombinant backcross. This chromosome contains the locus coding for mtRPOL, which has been the focus of recent studies of cytonuclear coadaptation in T. californicus. In comparison to our results, Ellison and Burton [22], looking at allelic frequencies in F4 hybrid adults, found evidence for selection against the SD mtRPOL genotype in crosses with both SC and another population, AB. They also observed that, unlike for other crosses, recombinant inbred lines with matching SD mtRPOL and SD mtDNA did not demonstrate the same OXPHOS transcriptional profile under conditions of hypo-osmotic stress as SD parentals, suggesting that an epistatically interacting nuclear locus is involved in mitochondrial transcription in SD. They suggested the transcription factor TFAM as a possible candidate. In this context, it is interesting that our results are suggestive of an epistatic interaction between marker TC167 and Chromosome 7; recent work (Rose and Edmands, unpublished) has revealed marker TC167 to be closely linked to TFAM. Nevertheless, the genotypic association patterns between mtRPOL and TC167 are not what would be expected if there is simple co-adaptation between SD mtRPOL and SD TFAM; individuals homozygous for SD mtRPOL exhibit a deficiency of SD homozygotes, and a slight excess of SC homozygotes, at TC167.

Higher divergence between parental lines is expected to result in increased frequencies of distorted loci [56], particularly due to heterozygote deficits [49]. For example, parental divergence is cited as the reason why crosses between mildly divergent $D$. pulex populations result in $21 \%$ of markers showing segregation distortion, largely due to homozygote deficits [49], while crosses between highly divergent Daphnia magna populations result in $33 \%$ of markers showing transmission ratio distortion, largely due to heterozygote deficits [44]. This pattern is consistent with the prediction that overdominance between alleles of closely related taxa may yield to underdominance between alleles in more distantly-related taxa [57]. Alternatively, divergence may increase the ratio of epistatic interactions involving heterozygous loci. In the current study hybridization between highly differentiated populations (over 20\% mitochondrial divergence $[15,16]$ ) led to a high frequency of marker distortion in adults (45\%), but no significant heterozygote deficits, indicating relatively slow accumulation of underdominance and/or epistasis involving heterozygotes.

As has been seen in other Tigriopus studies [30], and in other taxa [58] it is clear that nuclear loci can interact in a complex way to influence fitness; unfortunately we lack the power to investigate such interactions in more depth in the current study. Additionally, we did not consider epigenetic effects, which have previously been suggested to alter gene transcription in interpopulation hybrids of $T$. californicus [59]. Overall, our results suggest many intriguing avenues for further investigation into the genetic basis of reduced fitness in interpopulation hybrids of $T$. californicus. These studies will be greatly facilitated by the recent transcriptome assembly for both the SD and SC T. californicus populations [60], and by continuing advances in crustacean genomics [61].

\section{Conclusion}

We developed 45 population-diagnostic SNP markers for Tigriopus californicus, with which we generated the first linkage map available for the Copepoda. We used this to examine segregation distortion in F2 interpopulation hybrids, which are known to have reduced fitness compared to parental populations. We found dramatic segregation distortion in adult males, but not in newly hatched larvae, indicating that this distortion arises as a result of selection against particular genotypic combinations between hatching and adulthood. Distorted markers were not distributed randomly across the genome but clustered within particular linkage groups. In contrast to other studies, we found little evidence for cytonuclear co-adaptation in this interpopulation cross. Instead, different linkage groups exhibited markedly different patterns of distortion, that appear to have been influenced by nuclear-nuclear epistatic interactions and may also reflect genetic load carried within the parental lines.

\section{Additional material}

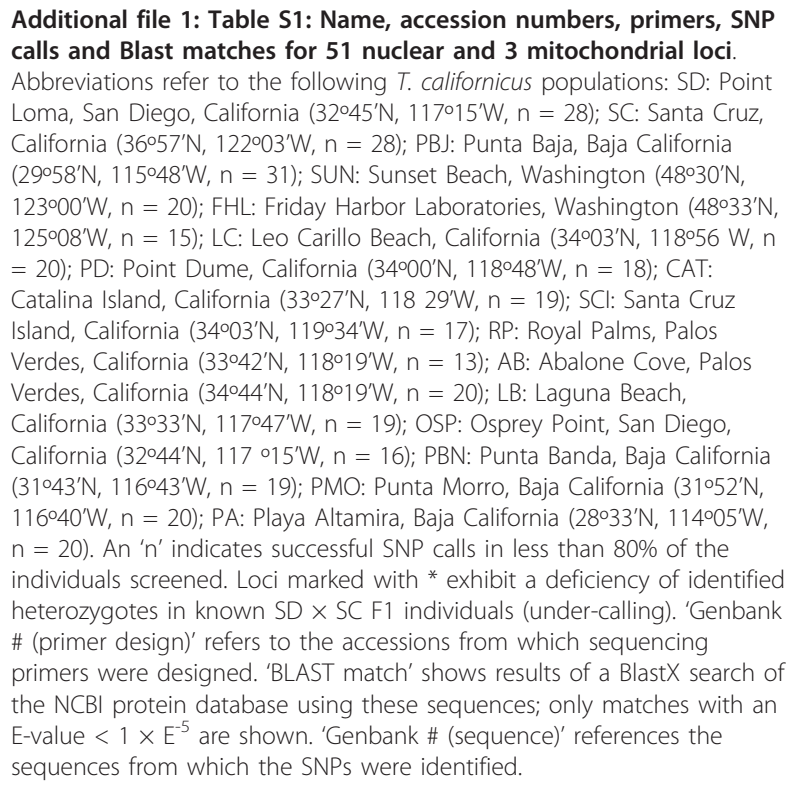


Additional file 2: Table S2: Number of inter-locus comparisions (n) map distance in CM Kosambi (Distance), standard error of map distance (SE), and LOD scores for all markers on the twelve chromosomes (Chrom). Data are shown for nauplii and adults, both including and omitting microsatellite markers. Total map lengths and map lengths corrected following $[43,44]$ are also provided.

\section{List of abbreviations}

ATP: adenosine triphosphate; CDNA: complementary deoxyribonucleic acid; CYC: cytochrome C; dNTP: deoxyribonucleotide triphosphate; LOD: logarithm of the odds; ME: malic enzyme; mRNA: messenger ribonucleic acid; PCR: polymerase chain reaction; QTL: quantitative trait locus; RISP: rieske ironsulfur protein.

\section{Acknowledgements and funding}

This work was funded by U.S. National Science Foundation Grants DEB0316807 to SE and DEB-0521176 to RSB. Undergraduate research students were supported by an NSF REU Site program (CCSV) and by funds from USC's Undergraduate Research Associates Program (JTZ) and Provost's Fellowship Program (LD). We thank Jeffrey Conroy for his great help in SNP assay design and genotyping, Annie Hwang, Catherine Purcell, Helen Truong and Tigran Karamanukyan for laboratory assistance, and Colin Rose for sharing unpublished data.

\section{Author details}

'Department of Biological Sciences, University of Southern California, Los Angeles, California 90089-0371, USA. ${ }^{2}$ Marine Biology Research Division, Scripps Institution of Oceanography, University of California at San Diego, La Jolla, California 92093-0202, USA. ${ }^{3}$ Southwest Fisheries Science Center, 110 Shaffer Road, Santa Cruz, CA 95060-5730, USA. ${ }^{4}$ Department of Biology, Georgia Southern University, Statesboro, Georgia 30460-8042, USA.

\section{Authors' contributions}

VLP performed SNP development, Tigriopus crosses, microsatellite genotyping, statistical analyses, and wrote the manuscript. SE designed the study and assisted with crosses. JSH and RSB generated EST data. JTZ, LD and CCSV assisted with SNP development and microsatellite genotyping. SE, JSH and RSB contributed to the manuscript, which all authors approved prior to submission.

Received: 13 December 2010 Accepted: 3 June 2011

Published: 3 June 2011

\section{References}

1. Dobzhansky T: Studies on hybrid sterility. II. Localization of sterility factors in Drosophila pseudoobscura hybrids. Genetics 1936, 21:113-135.

2. Muller $\mathrm{HJ}$ : Isolating mechanisms, evolution, and temperature. Biol Symp 1942, 6:71-125.

3. Bikard D, Patel D, Le Metté C, Giorgi V, Camilleri C, Bennett MJ, Loudet O: Divergent evolution of duplicate genes leads to genetic incompatibilities within A. thalania. Science 2009, 323:623-626.

4. Brideau NJ, Flores HA, Wang J, Maheshwari S, Wang X, Barbash DA: Two Dobzhansky-Muller genes interact to cause hybrid lethality in Drosophila. Science 2006, 314:1292-1295.

5. McDaniel SF, Willis JH, Shaw AJ: The genetic basis of developmental abnormalities in interpopulation hybrids of the moss Ceratodon purpureus. Genetics 2008, 179:1425-1435.

6. Seidel HS, Rockman MV, Kruglyak L: Widespread genetic incompatibility in C. elegans maintained by balancing selection. Science 2008, 319:589-594.

7. Edmands S: Recombination in interpopulation hybrids of the copepod Tigriopus californicus: release of beneficial variation despite hybrid breakdown. J Hered 2008, 99:316-318.

8. Johansen-Morris AD, Latta RG: The fitness consequences of hybridization between ecotypes of Avena barbata: hybrid breakdown, hybrid vigor, and transgressive segregation. Evolution 2006, 60:1585-1595.

9. Rieseberg LH, Sinervo B, Linder CR, Ungerer MC, Arias DM: Role of genetic interactions in hybrid speciation: evidence from ancient and experimental hybrids. Science 1996, 272:741-745.
10. Schweitzer JA, Martinsen GD, Whitham TG: Cottonwood hybrids gain fitness traits of both parents: a mechanism for their long-term persistence? Am J Bot 2002, 89:981-991.

11. Turelli M, Orr HA: Dominance, epistasis and the genetics of postzygotic isolation. Genetics 2000, 154:1663-1679.

12. Burton RS: Genetic evidence for long term persistence of marine invertebrate populations in an ephemeral environment. Evolution 1997, 51:993-998.

13. Burton RS, Feldman MW: Population genetics of Tigriopus californicus. II. Differentiation among neighboring populations. Evolution 1981, 35:1192-1205.

14. Willett CS, Burton RS: Evolution of interacting proteins in the mitochondrial electron transport system in a marine copepod. Mol Biol Evol 2004, 21:443-453.

15. Willett CS, Ladner JT: Investigations of fine-scale phylogeography in Tigriopus californicus reveal historical patterns of populations divergence. BMC Evolutionary Biology 2009, 9:139-158.

16. Edmands S: Heterosis and outbreeding depression in interpopulation crosses spanning a wide range of divergence. Evolution 1999, 53:1757-1768.

17. Ganz H, Burton RS: Genetic differentiation and reproductive incompatibility among Baja California populations of the copepod Tigriopus californicus. Mar Biol 1995, 123:821-827.

18. Burton RS: Hybrid breakdown in developmental time in the copepod Tigriopus californicus. Evolution 1990, 44:1814-1822.

19. Edmands S, Feaman HV, Harrison JS, Timmerman CC: Genetic consequences of many generations of hybridization between divergent copepod populations. J Hered 2005, 96:114-123.

20. Ellison CK, Burton RS: Interpopulation hybrid breakdown maps to the mitochondrial genome. Evolution 2008, 62:631-638.

21. Burton RS: Evolutionary consequences of restricted gene flow in the intertidal copepod Tigriopus californicus. Bull Mar Sci 1986, 39:526-535.

22. Ellison CK, Burton RS: Genotype-dependent variation of mitochondrial transcriptional profiles in interpopulation hybrids. Proc Natl Acad Sci 2008, 105:15831-15836.

23. Edmands S, Burton RS: Cytochrome c oxidase activity in interpopulation hybrids of a marine copepod: a test for nuclear-nuclear or nuclearcytoplasmic coadaptation. Evolution 1999, 53:1972-1978.

24. Ellison CK, Burton RS: Disruption of mitochondrial function in interpopulation hybrids of Tigriopus californicus. Evolution 2006 60:1382-1391.

25. Willett CS, Burton RS: Viability of cytochrome c depends on cytoplasmic background in Tigriopus californicus. Evolution 2001, 55:1592-1599.

26. Rawson PD, Burton RS: Functional coadaptation between cytochrome $c$ and cytochrome c oxidase within allopatric populations of a marine copepod. Proc Natl Acad Sci USA 2002, 99:12955-12958.

27. Harrison JS, Burton RS: Tracing hybrid incompatibilities to single amino acid substitutions. Mol Biol Evol 2006, 23:559-564.

28. Edmands S, Northrup SL, Hwang AS: Maladapted gene complexes within populations of the intertidal copepod Tigriopus californicus? Evolution 2009, 63:2184-2192.

29. Willett CS, Berkowitz JN: Viability effects and not meiotic drive cause dramatic departures from Mendelian inheritance for malic enzyme in hybrids of Tigriopus californicus populations. J Evol Biol 2007, 20:1196-1205.

30. Willett CS: Deleterious epistatic interactions between electron transport system protein-coding loci in the copepod Tigriopus californicus. Genetics 2006, 173:1465-1477.

31. Harrison JS, Edmands S: Chromosomal basis of viability differences in Tigriopus californicus interpopulation hybrids. J Evol Biol 2006, 19:2949-2051.

32. Edmands S, Harrison JS: Molecular and quantitative trait variation within and among populations of the intertidal copepod Tigriopus californicus. Evolution 2003, 57:2277-2285.

33. Burton RS: Mating system of the intertidal copepod Tigriopus californicus. Mar Biol 1985, 86:247-252.

34. Egloff DA: Ecological aspects of sex ratio and reproduction in experimental and field populations of the marine copepod Tigriopus californicus. PhD thesis, Stanford University 1966.

35. Vittor BA: Effects of the environment on fitness- related life history characters in Tigriopus californicus. PhD thesis, University of Oregon 1971. 
36. Burton RS: Differentiation and integration of the genome in populations of Tigriopus californicus. Evolution 1987, 41:504-513.

37. Ar-Rushdi $\mathrm{AH}$ : The cytology of achiasmatic meiosis in the female Tigriopus (Copepoda). Chromosoma 1963, 13:526.

38. Lazzaretto I, Libertini A: Karyological comparison among different Mediterranean populations of the genus Tigriopus (Copepoda Harpacticoida). Boll Zool 1986, 53:197-202.

39. Voordouw MJ, Anholt BR: Environmental sex determination in a splash pool copepod. Biol J Linn Soc 2002, 76:511-520.

40. Voordouw MJ, Anholt BR: Heritability of sex tendency in a harpacticoid copepod Tigriopus californicus. Evolution 2002, 56:1754-1763.

41. Willett CS: Significant variation for fitness impacts of ETS loci in hybrids between populations of Tigriopus californicus. J Heredity 2008, 99:56-65.

42. Harrison JS, Peterson DL, Swain JR, Edmands S: Microsatellite DNA markers for the intertidal copepod Tigriopus californicus. Mol Ecol Notes 2004, 4:736-738.

43. Chakravarti A, Lasher LK, Reefer JE: A maximum likelihood method for estimating genome length using genetic linkage data. Genetics 1991, 128:175-182

44. Routtu J, Jansen B, Colson I, De Meester L, Ebert D: The first generation Daphnia magna linkage map. BMC Genomics 2010, 11:508.

45. Niehuis O, Judson AK, Gadau J: Cytonuclear genic incompatibilities cause increased mortality in male F2 hybrids of Nasonia giraulti and $N$. vitripennis. Genetics 2008, 178:413-426.

46. Mauchline J: The Biology of Calanoid Copepods. Adv Mar Biol 1998, 33:1-709.

47. Hall MC, Willis JH: Transmission ratio distortion in intraspecific hybrids of Mimulus guttatus: implications for genomic divergence. Genetics 2005, 170:375-386.

48. Kuittinen H, De Haan AA, Vogl C, Oikarinen S, Leppälä J, Koch M, MitchellOlds T, Langley $\mathrm{CH}$, Savolainen O: Comparing the linkage maps of the close relatives Arabidopsis lyrata and A. thaliana. Genetics 2004, 168:1575-1584.

49. Critescu MEA, Colbourne JK, Radivojac J, Lynch M: A microsatellite-based genetic linkage map of the waterflea, Daphnia pulex: On the prospect of crustacean genomics. Genomics 2006, 88:415-430.

50. López-Fernández H, Bolnick Dl: What causes partial F1 hybrid viability? Incomplete penetrance versus genetic variation. PLOS ONE 2007, 2:e1294.

51. Launey S, Hedgecock D: High genetic load in the Pacific oyster Crassostrea gigas. Genetics 2001, 159:255-265.

52. Martin NH, Bouck AC, Arnold ML: Loci affecting long-term hybrid survivorship in Louisiana irises: implications for reproductive isolation and introgression. Evolution 2005, 59:2116-2124.

53. Rogers SM, Bernatchez $L$ : The genetic basis of intrinsic and extrinsic postzygotic reproductive isolation jointly promoting speciation in the lake whitefish species complex (Coregonus clupeaformis). J Evol Biol 2006 19:1979-1994.

54. Willett CS, Burton RS: Environmental influences on epistatic interactions: viabilities of cytochrome c genotypes in interpopulation crosses. Evolution 2003, 57:2286-2292

55. Fishman L, Kelly AJ, Morgan E, Willis JH: A genetic map in the Mimulus guttatus species complex reveals transmission ratio distortion due to heterospecific interactions. Genetics 2001, 159:1701-1716.

56. Jenczewski E, Gherardi M, Bonnin I, Prosperi JM, Olivierei I, Huguet T: Insight on segregation distortions in two intraspecific crosses between annual species of Medicago (Leguminosae). Theor Appl Genet 1997, 94:682-691.

57. Lynch $M$ : The genetic interpretation of inbreeding depression and outbreeding depression. Evolution 1991, 45:622-629.

58. Moyle LC, Nakazato T: Complex epistasis for Dobzhansky-Muller hybrid incompatibility in Solanum. Genetics 2009, 181:347-351.

59. Flowers JM, Burton RS: Ribosomal RNA gene silencing in interpopulation hybrids of Tigriopus californicus: nucleolar dominance in the absence of intergenic spacer subrepeats. Genetics 2006, 173:1479-1486.

60. Barreto FS, Moy GW, Burton RS: Interpopulation patterns of divergence and selection across the transcriptome of the copepod Tigriopus californicus. Mol Ecol 2010, 20:560-572.

61. Colbourne JK, Pfrender ME, Gilbert D, et al: The ecoresponsive genome of Daphnia pulex. Science 2011, 331:555-561 doi:10.1186/1471-2156-12-54

Cite this article as: Pritchard et al:: Interpopulation hybridization results in widespread viability selection across the genome in Tigriopus californicus. BMC Genetics 2011 12:54.

\section{Submit your next manuscript to BioMed Central and take full advantage of:}

- Convenient online submission

- Thorough peer review

- No space constraints or color figure charges

- Immediate publication on acceptance

- Inclusion in PubMed, CAS, Scopus and Google Scholar

- Research which is freely available for redistribution

Submit your manuscript at www.biomedcentral.com/submit
Biomed Central 